\title{
Robotic guidance platform for laser interstitial thermal ablation and stereotactic needle biopsies: a single center experience
}

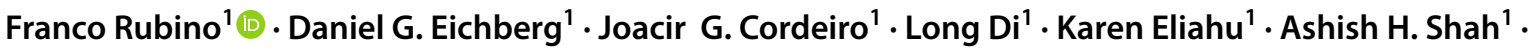 \\ Evan M. Luther ${ }^{1} \cdot$ Victor M. Lu $^{1} \cdot$ Ricardo J. Komotar ${ }^{1,2} \cdot$ Michael E. Ivan $^{1,2}$
}

Received: 1 February 2021 / Accepted: 4 July 2021 / Published online: 13 July 2021

(c) The Author(s), under exclusive licence to Springer-Verlag London Ltd., part of Springer Nature 2021

\begin{abstract}
While laser ablation has become an increasingly important tool in the neurosurgical oncologist's armamentarium, deep seated lesions, and those located near critical structures require utmost accuracy during stereotactic laser catheter placement. Robotic devices have evolved significantly over the past two decades becoming an accurate and safe tool for stereotactic neurosurgery. Here, we present our single center experience with the MedTech ROSA ONE Brain robot for robotic guidance in laser interstitial thermal therapy (LITT) and stereotactic biopsies. We retrospectively analyzed the first 70 consecutive patients treated with ROSA device at a single academic medical center. Forty-three patients received needle biopsy immediately followed by LITT with the catheter placed with robotic guidance and 27 received stereotactic needle biopsy alone. All the procedures were performed frameless with skull bone fiducials for registration. We report data regarding intraoperative details, mortality and morbidity, diagnostic yield and lesion characteristics on MRI. Also, we describe the surgical workflow for both procedures. The mean age was $60.3 \pm 15$ years. The diagnostic yield was positive in $98.5 \%(n=69)$. Sixty-three biopsies (90\%) were supratentorial and seven (10\%) were infratentorial. Gliomas represented $54.3 \%$ of the patients $(n=38)$. There were two postoperative deaths (2.8\%). No permanent morbidity related to surgery were observed. We did not find intraoperative technical problems with the device. There was no need to reposition the needle after the initial placement. Stereotactic robotic guided placement of laser ablation catheters and biopsy needles is safe, accurate, and can be implemented into a neurosurgical workflow.
\end{abstract}

Keywords Robotic neurosurgery $\cdot$ LITT $\cdot$ Stereotactic procedures $\cdot$ Brain tumors $\cdot$ ROSA robot

\section{Introduction}

In the last quarter of the century, the successful use of industrial robotics for highly specific, highly precise, and dangerous tasks stimulated renewed interest in transferring these concepts to the medical field [1]. The robots have had the potential to deliver faster results than any other procedures available that required the manual adjustment of the stereotactic frame because the robots' computer calculates and make adjustments faster than humans [2]. The recently

Franco Rubino

franco_rubino_007@ hotmail.com

1 Department of Neurological Surgery, University of Miami Miller School of Medicine, Lois Pope Life Center, 1095 NW 14th Terrace (D4-6), Miami, FL 33146, USA

2 Sylvester Comprehensive Cancer Center, Miami, FL 33146, USA
FDA-approved Robotic Surgical Assistant (ROSA ONE Brain; MedTech, France) is an emerging robotic technology used in neurological surgery to assist in planning and performing minimally invasive procedures. While use of ROSA in the epilepsy field has been described extensively for stereo-electroencephalography (SEEG) placement [3, 4] and laser ablation of epileptogenic foci $[5,6]$ data regarding its utility in neurosurgical oncology is limited.

In neuro-oncology, safe and accurate placement of stereotactic biopsy needles and laser ablation catheters is critical with the goal of reducing morbidity and mortality through the preservation of eloquent brain areas and avoidance of vasculature [7]. Laser interstitial thermal therapy (LITT) has become an increasingly important tool in the neurosurgical oncologist's armamentarium [8, 9]. However, deep seated lesions, and those located near critical structures require utmost accuracy during stereotactic laser catheter placement. 
The data regarding the utility of robotic guidance for LITT are limited.

In this study, we present a large series of patients (27 biopsies and 43 MRI-guided LITT) surgically treated with the assistance of the ROSA ONE Brain robot. We describe our results, surgical technique and workflow for using this system.

\section{Methods}

\section{Ethics}

Institutional review board approval was obtained for this study. The consent process was waived, because this was a retrospective study stripped of all identifying patient information. The surgeons in the intraoperative pictures consented to publication.

\section{Study design, setting and patients}

This retrospective and single center study was conducted in accordance with PROCESS guidelines [10]. The patients were surgically treated at University of Miami Hospital from March 2019 to August 2020. The ROSA ONE Brain robot was acquired by our institution on March 2019 and, since then, all the stereotactic procedures have been made with this FDA-approved platform. Surgeries were performed by staff surgeons and assisted by residents.

The electronic medical records were read for data abstraction (Table 1). The number of successful biopsies was defined as the no need for a repeat biopsy due to insufficient tissue collection. Tumor location was divided in supratentorial and infratentorial. Supratentorial tumors were subdivided into three groups: superficial tumors (located up to $2 \mathrm{~cm}$ from the cortical surface), deep tumors (located more than $2 \mathrm{~cm}$ far away from the cortical surface) and tumors
Table 1 Statistical data from "Needle biopsy" group and

"Laser ablation" group

\begin{tabular}{|c|c|c|}
\hline \multirow{2}{*}{$\begin{array}{l}\text { Characteristic } \\
\text { Type of robotic-assisted procedure }\end{array}$} & \multicolumn{2}{|l|}{ Value $(\%)$} \\
\hline & Needle biopsy (27) & Laser ablation (43) \\
\hline Age (years) & $59.3 \pm 17.75$ & $60.9 \pm 12.7$ \\
\hline \multicolumn{3}{|l|}{ Number of patients $(\mathrm{N})$} \\
\hline Male-to-female ratio & $0.8: 1$ & $0.65: 1$ \\
\hline Male & $12(44.4 \%)$ & $17(39.5 \%)$ \\
\hline Female & $15(55.6 \%)$ & $26(60.5 \%)$ \\
\hline \multicolumn{3}{|l|}{ Pathology result } \\
\hline Diagnostic & $27(100 \%)$ & $42(97.6 \%)$ \\
\hline Gliomas & $15(55.6 \%)$ & $23(53.6 \%)$ \\
\hline Metastatic tumors & $3(11.1 \%)$ & $3(6.9 \%)$ \\
\hline Lymphoma & $2(7.4 \%)$ & - \\
\hline Autoimmune disease & $1(3.7 \%)$ & - \\
\hline Demyelinization disease & $1(3.7 \%)$ & - \\
\hline Radiation Necrosis & $2(7.4 \%)$ & $10(23.3 \%)$ \\
\hline Gliosis/Necrosis & $3(11.1 \%)$ & $6(13.9 \%)$ \\
\hline Non-diagnostic & $0(0.0 \%)$ & $1(2.3 \%)$ \\
\hline \multicolumn{3}{|l|}{ Location } \\
\hline Supratentorial & $24(88.8 \%)$ & $39(90.6 \%)$ \\
\hline Superficial tumors & $6(22.2 \%)$ & $7(16.2 \%)$ \\
\hline Deep tumors & $14(51.8 \%)$ & $24(55.8 \%)$ \\
\hline Corpus callosal & $4(14.8 \%)$ & $8(18.6 \%)$ \\
\hline Infratentorial & $3(11.2 \%)$ & $4(9.4 \%)$ \\
\hline Volume $\left(\mathrm{cm}^{3}\right)$ & $5.9 \pm 5.9$ & $9.2 \pm 13.1$ \\
\hline Number of needle reposition & $0(0 \%)$ & $0(0 \%)$ \\
\hline Number of reoperation & $0(0 \%)$ & $1(2.3 \%)$ \\
\hline Technical problems with robot & $0(0 \%)$ & $0(0 \%)$ \\
\hline Time of the procedure (min) & $75.2 \pm 40$ & $100.3 \pm 47$ \\
\hline \multicolumn{3}{|c|}{ Postoperative complication (Clavien-Dindo) } \\
\hline Grade I & $1(3.7 \%)$ & $5(11.6 \%)$ \\
\hline Grade V & $2(7.4 \%)$ & $0(0 \%)$ \\
\hline
\end{tabular}


arising from the corpus callosum. The tumor volume was calculated using the ellipsoid formula [11]. Duration of the procedure was measure in minutes, in needle biopsies, we considered the time from the skin incision to the closure, and in LITT cases, we consider from the skin incision to the beginning of the intraoperative MRI. The time for trajectory planning was not considered, because it is performed the night before the procedure.

We used descriptive statistic to analyze our results. Categorical variables were presented as proportions and the continuous variables were presented with mean and standard deviation. The data were analyzed using GraphPad Prism 6 (GraphPad Software, USA).

\section{Surgical technique and workflow}

\section{Preoperative considerations}

Trajectories are planned the day before using a preoperative thin slice MRI. SWI-MRI sequence is also obtained to help avoid trajectories that lead to tumor areas that have bled previously, and may be at higher risk for bleeding intraoperatively (Fig. 1A, B). The planned trajectory should avoid all eloquent regions, vessels, sulci, and ventricles down the center of the lesion (Fig. 2A). If a laser ablation will be performed, the trajectory is planned along the long axis of the lesion.

\section{Patient positioning considerations}

Almost all biopsies and laser ablations can be performed with the patient supine, with a shoulder bump and head turn as needed. In our experience, biopsies and laser ablations of midline cerebellar lesions should be performed prone. However, all other lesions, including mesial temporal lesions accessed from a posterior to anterior trajectory, can be safely performed in a supine position.

\section{Patient registration}

The patient is intubated and sedated. The first step consists in placement of the skull bone fiducials for registration. In the supine position, five bone fiducials are placed throughout the skull without fixation of the head in a skull clamp (Fig. 2B, C). If the patient is in the prone position, the bone fiducials are placed after the head is fixed with a skull clamp.

After the bone fiducials are placed, an intraoperative thin slice O-arm CT scan is performed (Fig. 2D), and then merged with the preoperative MRI. The patient's head is then secured with a skull clamp and placed in the final surgical position. The head clamp is affixed to the ROSA robot (Fig. 2E). To avoid inadvertent movement while the patient is affixed to the ROSA robot, the OR bed control should be disconnected. Afterwards, a pointer probe is attached to the robot arm for registration. Registration is performed by bringing the ROSA pointer probe to touch each bone fiducial and then confirmed on multiple anatomic points (Fig. 2F). The ROSA arm can be used to identify the planned trajectory entry point, which is marked on the skin with a marker.

It should be noted that the robot is equipped with a registration system that combines robotic movements with noninvasive touch-free laser measurement for frameless surface registration [12].
Fig. 1 Preoperative images. Axial brain MRI with contrast showing a big right insular tumor with heterogenous enhancement (A). Preoperative susceptibility weighted-image MRI (SWI) is strongly recommended to identify areas of high tumoral vascularization or previous bleeding to avoid these areas in the planned trajectory (B)
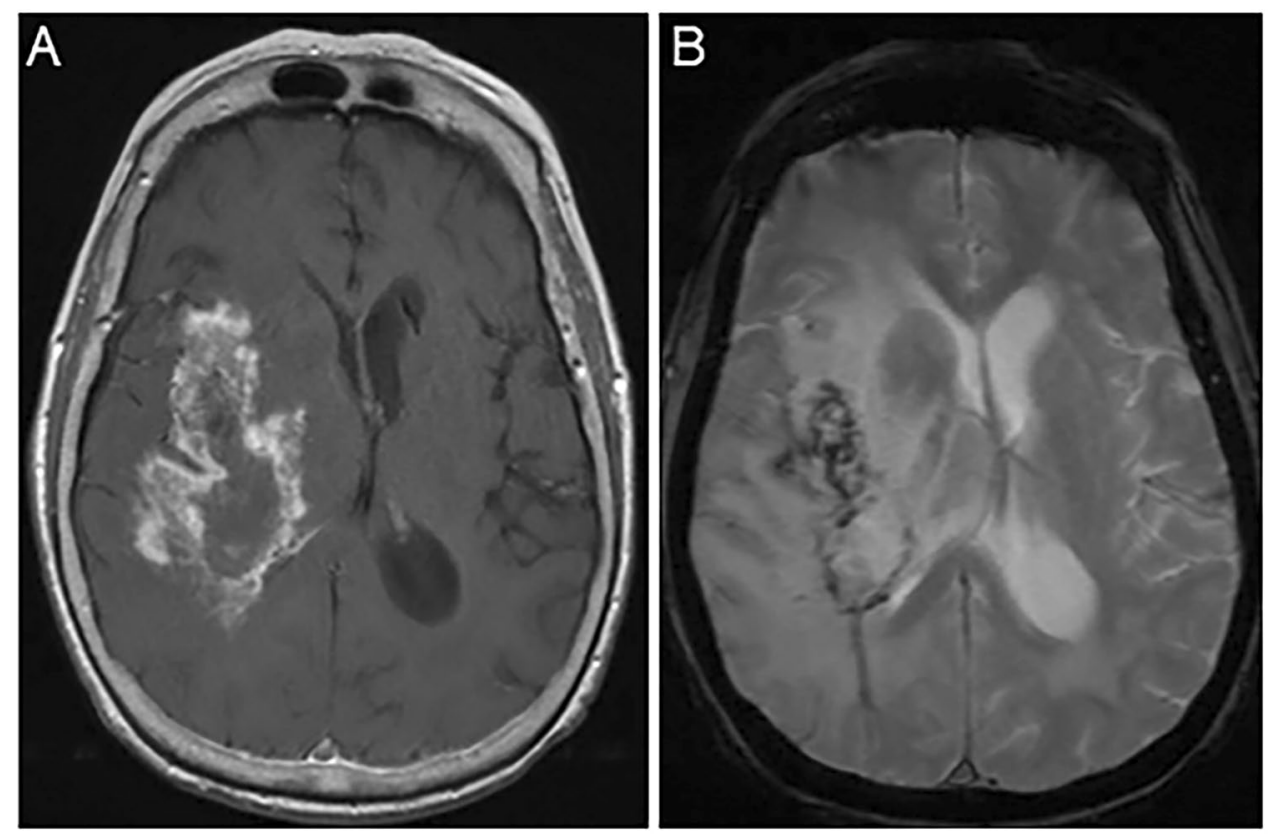

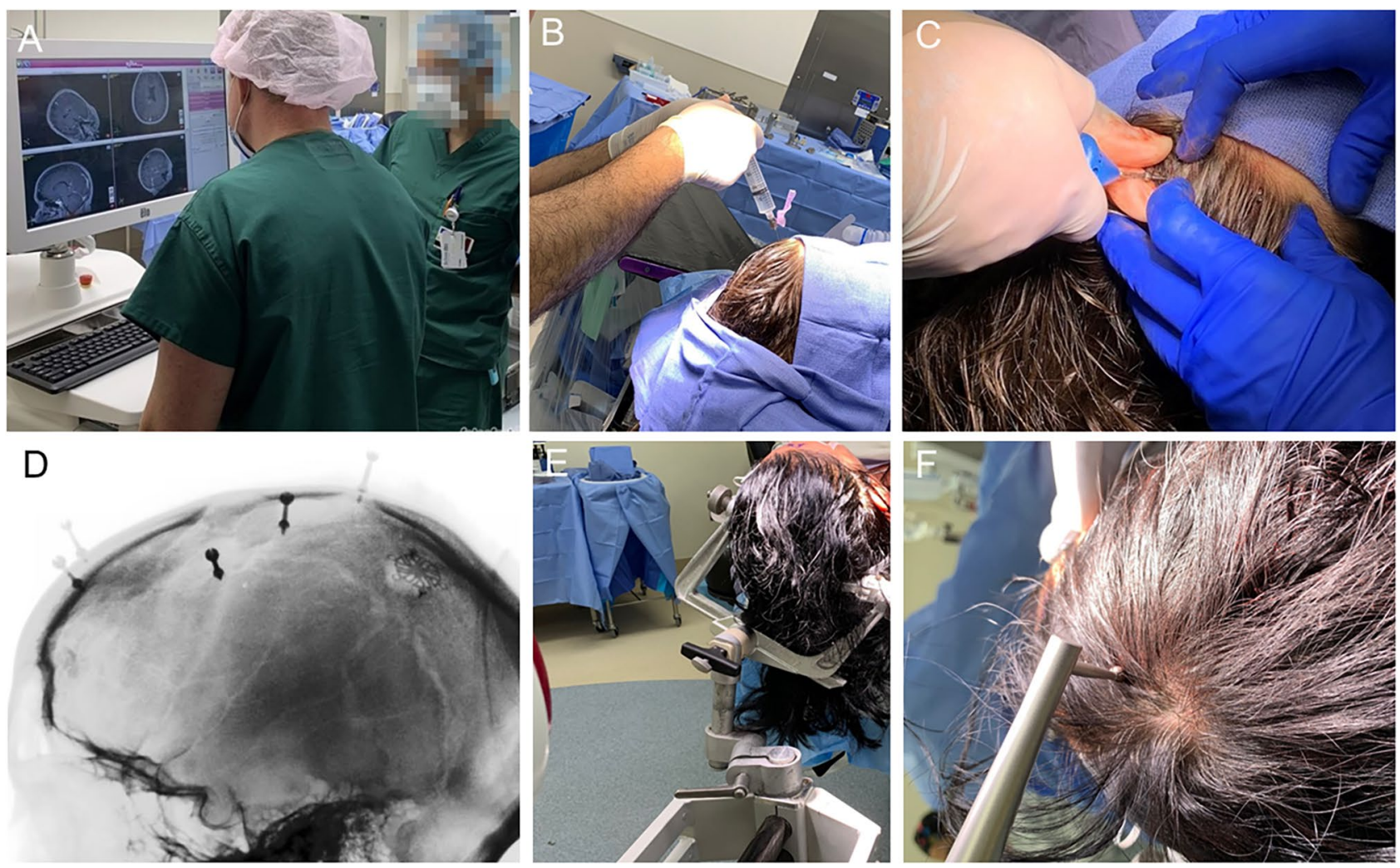

Fig. 2 Preoperative surgical planning with ROSA platform. Using the touch screen, the surgical team register in the platform the trajectory planned the day before and configurate a needle route to the target (A). After this step, local anesthetic is injected (B) and skull fiducials must be placed (C). Next step consists of getting a high-resolution fine-cut intraoperative CT (in our case we use the O-arm, Medtronic). Bone fiducials are placed in preoperative holding in a pattern distributed over both hemispheres (D). The CT is used for matching intraoperative and preoperative planning data on ROSA software interface.
The patient's head is then secured with a skull clamp, the patient is placed in the final surgical position, and the head clamp is affixed to the ROSA robot $(\mathbf{E})$. The ROSA robot's wheels are locked in place, and the OR table bed control is disconnected from the bed to avoid inadvertent movement of the OR table, while the patient is affixed to the ROSA robot. A pointer probe is attached to ROSA arm. Then, registration is performed by bringing a pointer probe to touch each bone fiducial and then confirmed on multiple anatomic points $(\mathbf{F})$

\section{Surgical technique}

Needle biopsy The patient is prepared and draped in standard fashion. The ROSA robot is engaged and the arm is used to identify the entry point. Once the entry point is chosen, a small stab incision is made. The ROSA arm is used to align a power drill to make as small burr hole. The dura is opened sharply using a $\mathrm{K}$ wire. After the dural opening and using the robotic frameless neuronavigation, the guidance rod from the arm is placed above the burr hole. Next, the ROSA computer calculates the depth to the target, which is marked on the Nashold needle (Nashold, Integra, USA). The ROSA arm is used to align the needle in the correct trajectory (Fig. 3A), and is then introduced to the target point (Fig. 3B). The samples are taken in four quadrants. The needle is removed and the stab incision is closed with an interrupted absorbable suture. The drapes and the bone fiducials are removed.

LITT For patients that undergo LITT, we use Visualase Thermal Therapy System (Medtronic, Minnesota, USA) [13]. Following the stereotactic needle biopsy, ROSA arm is used to align the placement of a guidance bolt (Fig. 3C), which is securely affixed into the skull. The ROSA computer calculates the depth to the target. A $1.8 \mathrm{~mm}$ laser cannula is placed through the alignment rod and the skull bolt (Fig. 3D). After alignment, the cannula length trajectory is marked in the laser catheter and the precision aiming rod device is moved out of the way. The laser catheter is progressed through the guidance bolt to the lesion. There should be no resistance (Fig. 3E). The bolt and catheter are secured and wrapped with Xeroform (Fig. 3F). The head is detached from the skull clamp, the patient transferred onto a stretcher and brought down to MRI for the second part of the procedure.

The MRI reference images are obtained and the laser catheter is confirmed to be in optimal position. The treatment 

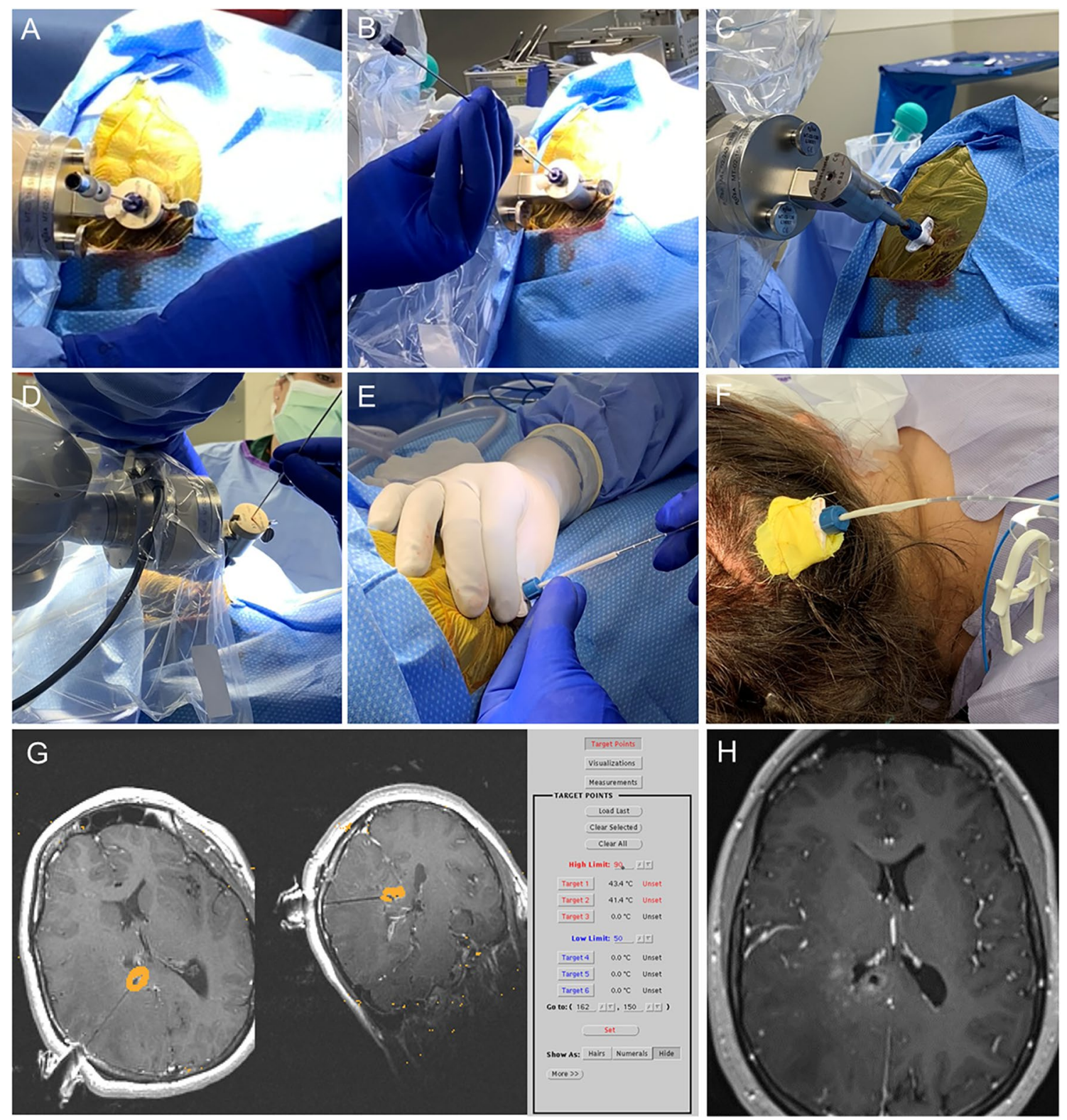

Fig. 3 Surgical workflow. Before drilling, the alignment error must be taken at least 3 times and this error might be less than $1 \mathrm{~mm}$. After defining the entry point, a small stab incision and a twist-drill burr hole are made. The dura is opened sharply using an angiocath or a $\mathrm{K}$ wire. Next, the alignment rod from the robot is placed above the burr hole. A The biopsy needle (Nashold, Integra, USA) is advanced through the rod into the lesion and tissue samples are sent off for permanent pathology. B When biopsy is completed, the needle is removed. These two steps represent the biopsy needle procedure. For patients that undergo LITT procedure, we have to move away the alignment rod and screwed the skull guidance bolt from the laser system. We use Visualase Thermal Therapy System (Medtronic).

When the skull bolt is placed, the alignment rod is repositioned above the bolt and locked (C). A $1.8 \mathrm{~mm}$ reducing laser cannula is placed through the alignment rod and the drilled hole. The cannula length trajectory is marked in the laser catheter $(\mathbf{D})$ and the precision aiming rod device is moved out of the way. The laser catheter is progressed through the guidance bolt to the lesion (E). At this point, the bolt and catheter are secured and wrapped with Xeroform $(\mathbf{F})$ and the patient brought down to MRI room for the second part of the procedure. An MRI reference images are obtained, and the laser catheter is checked to be in the optimal position for the laser ablation technique $(\mathbf{G})$. A postoperative MRI confirm a successful ablation $(\mathbf{H})$

plan is created and ablation is performed (Fig. 3G). After the ablation is completed (Fig. $3 \mathrm{H}$ ), the laser catheter is removed and the stab incision is closed with a single absorbable suture. 


\section{Results}

\section{Patient demographics}

The male-to-female ratio was $0.71: 1$. The mean age was $60.3 \pm 15$ years. Forty-three patients $(61.4 \%)$ received laser ablation and 27 patients only needle biopsy (38.6\%). All the laser procedures included a biopsy before the ablation. The age and sex distribution were similar between both groups (Table 1).

\section{Tumor pathology, location and volume}

Tumor pathology included gliomas from low-grade to high-grade gliomas and glioblastomas $(n=38,54.3 \%)$, lymphoma ( $n=2,2.9 \%)$, metastatic tumors $(n=6,8.6 \%)$, radiation necrosis $(n=12,17.2 \%)$, autoimmune encephalitis $(n=1,1.4 \%)$, demyelinization disease $(n=1,1.4 \%)$, gliosis or necrosis $(n=9,12.8 \%)$ and nondiagnostic or inconclusive results $(n=1,1.4 \%)$.

In terms of location $90 \%(n=63)$ of the lesions were supratentorial and $10 \%(n=7)$ were infratentorial. Five of the infratentorial tumors were in the cerebellum and two were in the brain stem (Table 1).

The mean volume of the lesions was $7.9 \pm 11 \mathrm{~cm}^{3}$. The smallest lesion was $0.15 \mathrm{~cm}^{3}$ and the biggest one was $65.2 \mathrm{~cm}^{3}$.

\section{Intervention compliance, tolerability and postoperative complications}

In a laser ablation procedure, we evaluate the optimal position of the laser catheter with an intraoperative MRI. None of the patients from laser group required a new reposition of the needle. The precision to place the catheter was successful in all the patients (Table 1).

Only one patient $(1.6 \%)$ needed to be operated again because of inconclusive result in pathology. During the procedure, the robotic system worked in $100 \%$ of cases and there was no need to change to another stereotactic system because of technical problems. Duration of the procedure were $75.2 \pm 40$ and $100.3 \pm 47 \mathrm{~min}$ in the needle biopsy group and LITT group, respectively.

Most of the patients were discharged on postoperative day $1(n=65,93 \%)$. Three patients were discharged on postoperative day $2(4.2 \%)$ and two patients died from an acute complication after the procedure (2.8\%). Among dead patients, one had a massive GBM hemorrhage in the second day after surgery (Fig. 4A). The other patient had a posterior fossa hematoma from a metastatic melanoma in the first postoperative day (Fig. 4B).

According to the Clavien-Dindo classification [14], the reported grades of complication were I and V in $6(9.7 \%)$ and $2(2.9 \%)$ patients, respectively. The therapeutics regimens applied for grade I patients were antiemetic and analgesic. No wound complications were found in this series allowing for early radiation treatment as per neurooncological indications in oncologic patients.
Fig. 4 Images from the two patients with postoperative hemorrhages. Patient with right thalamic GBM who died in the second postoperative day (A). Patient with right inferior cerebellar peduncle melanoma metastasis who died in the first postoperative day (B)
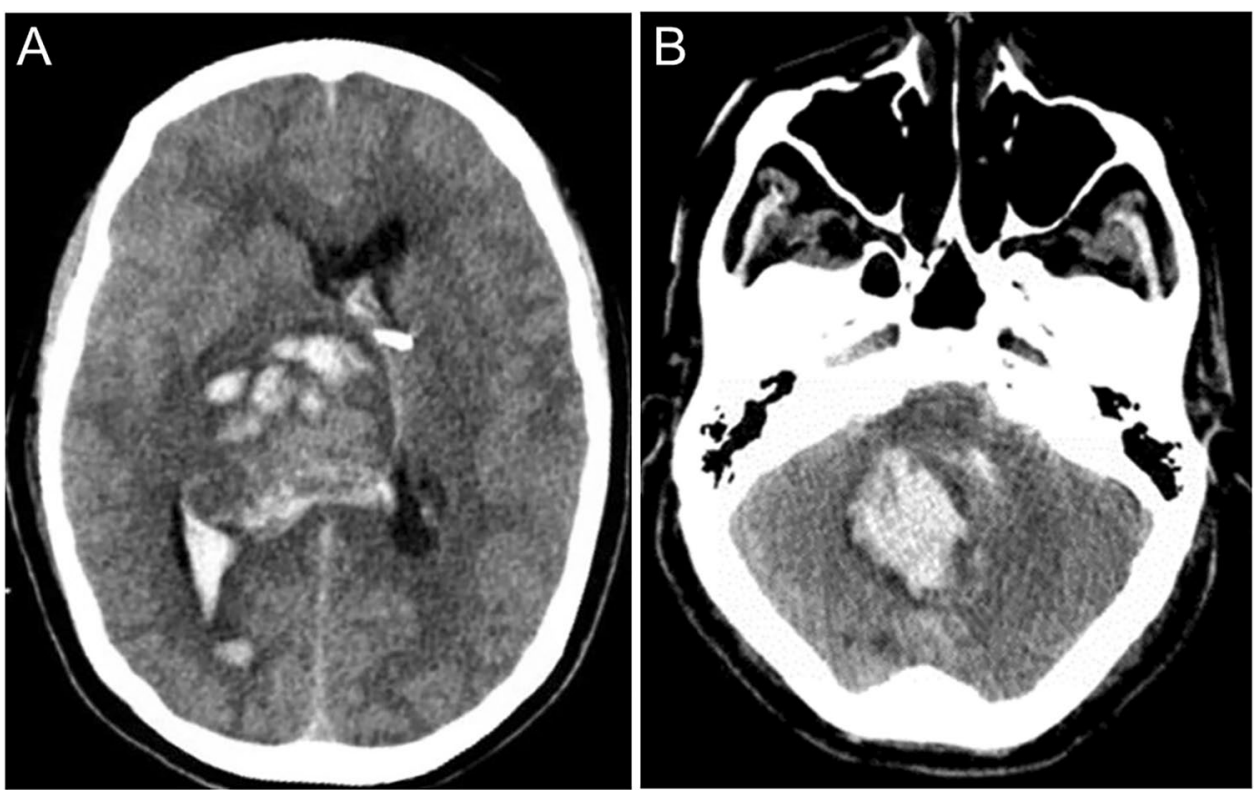


\section{Discussion}

This report represents one of the largest series in roboticassisted cranial procedures $[12,15-18]$, and, to our knowledge, this is the largest clinical series with the use of Robotic Surgical Assistant system (ROSA ONE Brain; MedTech, France) for laser ablation procedures, since its introduction [19]. According to our results, the ROSA robotic platform had an excellent performance in terms of accuracy and safety and there was no need for needle repositions or technical problems during the procedures. We successfully performed 27 stereotactic biopsies and 43 biopsies followed by MRI-guided laser interstitial ablation of metastasis and primary malignant tumors.

Stereotactic procedures are among the most frequently performed procedures in cranial neurosurgery. There are well-known differences between frame-based, frameless, or even free-hand techniques [12, 16, 20, 21]. Framebased procedures are still the gold standard when comparing frameless and robotic techniques [20, 22]. In fact, some authors found that the frameless technique has less accuracy with larger spread of values in the planned target, especially in implanted DBS electrodes [20, 23, 24]. To solve these slight differences, robotic precision has been increasingly utilized in the operating room in the last decade.

Robotics allow for accurate, safe, frameless, and rapid procedures by combining the advantages of both frameless and frame-based procedure [12]. In our series, the rate of nondiagnostic biopsy was $1.4 \%(n=1)$. This rate is lower than other robotic series which report between 2.2 and $4.5 \%$ of nondiagnostic results $[12,25]$. Regarding to lesion size, some authors suggest a minimum size $(15 \mathrm{~mm})$ for the frameless procedure [21, 26, 27]. Seventeen patients in our series had lesions with a diameter smaller than $15 \mathrm{~mm}$ and the diagnostic rate was successful in $100 \%$ of these patients. To highlight the accuracy of the robotic procedure, 11 out of these 17 patients received MRI-guided LITT and the needle was always in an optimal position in the intraoperative MRI. According to this, the minimum size for a lesion to be biopsied using a frameless equipment should be revised in robotic procedures.

In terms of safety, surgical robots are constantly evaluated and over the years, they gain sophisticated technological progress to minimize the patient harm risk. This is the main topic to justify the highly increased cost [28] of introducing this robotic platforms in the operating room. In our series, no infectious morbidity or permanent sequelae related to the procedure were observed. We presented only two deaths after the biopsy but these complications were likely not caused specifically by use of the ROSA robot, but rather the inherent risk of performing a stereotactic needle biopsy on these types of tumors. Metastatic melanoma and high-grade gliomas are lesions with high risk of bleeding [29]. The general percentage of mortality is similar to other non-robotic large series in terms of morbidity and mortality [30-33].

In all robotic platforms is crucial understanding the performance profiles. According to our experience, the ROSA robot had successfully accomplished the vast majority of the procedures without any technical issue, and the system proved to be highly stable throughout the procedures. None of the patients needed a needle reposition. Moreover, classical trajectories considered as "extreme locations" in frame-based techniques, like the lower temporal lobe and posterior fossa were feasible to approach [16]. Before our practice used the ROSA robot, we used the Medtronic Stealth Station, Vertek Arm system. While we had favorable results with the Vertek Arm system, the learning curve is quite steep, as the trajectory alignment requires securing 7 degrees of freedom. This process can be quite difficult, even for experienced surgeons. In our experience, when using a single trajectory, the Vertek Arm system is faster than the ROSA robot, as the ROSA robot takes longer to register to the patient. However, when using multiple trajectories during a single procedure, the ROSA robot is faster.

Another advantage of ROSA platform is that this device can be used to perform all types of registration: frame-based, frameless fiducial marker (skin or bone), and frameless surface registration [12]. There is no accuracy benefit of bone fiducials over laser facial scanning [34]. Furthermore, the robot is easily combined with intraoperative images and the needle in position can be coregistered with preoperative planning images, allowing the surgeon to confirm that the needle is accurately placed [19].

Among the disadvantages of this platform, we could mentioned the dimensions of the device, the learning curve and the high initial cost $[28,35]$. In the operating room, the robot must be placed relatively close to the patient's head and occupies a space almost equivalent to that of a human being in the operative field, so the presence of more than one surgeon would be uncomfortable. The software interface of the platform is relatively easy to use and very similar to other neuronavigational systems and the most crucial part is how to manage the robotic arm. One interesting point to mention in our experience is that during the flight restrictions with COVID-19 pandemic, the technician could not travel to assist with the use of the platform. In the future, remote assistance could solve this issue.

Finally, the high initial cost of ROSA platform could be overwhelmed with its multiple applications not only limited to stereotactic biopsies [4, 15, 36]. Future studies should focus in the statistical analysis to find differences among frameless robotic and frameless non-robotic procedures 
to justify the cost-effectiveness of the device to be used in countries with limited resources.

\section{Conclusion}

We presented the largest series of stereotactic laser ablation with the ROSA robot. ROSA robot can successfully and safely be used for stereotactic biopsies and laser ablation therapy. To our experience, the platform was user-friendly and simple to use and has a short intraoperative setup for the registration of the patient and the planned trajectory. The accuracy and precision for stereotactic targeting were successful with only one-time needle placement.

Acknowledgements We would like to acknowledge Corinne Gorant for his assistance in proofreading the manuscript.

Funding Franco Rubino MD is part of the International Observership Program (IOP), a program supported by the CNS Foundation.

\section{Declarations}

Conflict of interest The author Michael E. Ivan is a consultant for Medtronic Corp. The reminder authors, Franco Rubino; Daniel G. Eichberg; Joacir G. Cordeiro, Long Di, Karen Eliahu; Ashish H. Shah; Evan M. Luther; Victor M. Lu; Ricardo J. Komotar declare that they have no conflict of interest.

\section{References}

1. Kwoh YS, Hou J, Jonckheere EA, Hayati S (1988) A robot with improved absolute positioning accuracy for CT guided stereotactic brain surgery. IEEE Trans Biomed Eng. https://doi.org/10.1109/ 10.1354

2. Doulgeris JJ, Gonzalez-Blohm SA, Filis AK et al (2015) Robotics in neurosurgery: evolution, current challenges, and compromises. Cancer Control. https://doi.org/10.1177/107327481502200314

3. Zhao R, Xue P, Zhou Y et al (2020) Application of robot-assisted frameless stereoelectroencephalography based on multimodal image guidance in pediatric refractory epilepsy: experience of a pediatric center in a developing country. World Neurosurg. https:// doi.org/10.1016/j.wneu.2020.04.218

4. Ho AL, Muftuoglu Y, Pendharkar AV et al (2018) Robot-guided pediatric stereoelectroencephalography: single-institution experience. J Neurosurg Pediatr. https://doi.org/10.3171/2018.5.PEDS1 7718

5. Fayed I, Sacino MF, Gaillard WD et al (2018) MR-guided laser interstitial thermal therapy for medically refractory lesional epilepsy in pediatric patients: experience and outcomes. Pediatr Neurosurg. https://doi.org/10.1159/000491823

6. Gonzalez-Martinez J, Vadera S, Mullin J et al (1982) Robotassisted stereotactic laser ablation in medically intractable epilepsy: operative technique. Neurosurgery 10:167-172. https://doi. org/10.1227/NEU.0000000000000286

7. Mattei TA, Rodriguez AH, Sambhara D, Mendel E (2014) Current state-of-the-art and future perspectives of robotic technology in neurosurgery. Neurosurg Rev 37(3):357-366. https://doi.org/10. 1007/s10143-014-0540-z

8. Shah AH, Semonche A, Eichberg DG et al (2020) The role of laser interstitial thermal therapy in surgical neuro-oncology: series of 100 consecutive patients. Neurosurgery. https://doi. org/10.1093/neuros/nyz424

9. Eichberg DG, VanDenBerg R, Komotar RJ, Ivan ME (2018) Quantitative volumetric analysis following magnetic resonanceguided laser interstitial thermal ablation of cerebellar metastases. World Neurosurg. https://doi.org/10.1016/j.wneu.2017.11. 098

10. Agha RA, Fowler AJ, Rajmohan S et al (2016) Preferred reporting of case series in surgery; the PROCESS guidelines. Int J Surg. https://doi.org/10.1016/j.ijsu.2016.10.025

11. Opalak CF, Parry M, Rock AK et al (2019) Comparison of ABC/2 estimation and a volumetric computerized method for measurement of meningiomas using magnetic resonance imaging. J Neurooncol. https://doi.org/10.1007/s11060-019-03205-z

12. Lefranc M, Capel C, Pruvot-Occean AS et al (2015) Frameless robotic stereotactic biopsies: a consecutive series of 100 cases. J Neurosurg. https://doi.org/10.3171/2014.9.JNS14107

13. Torres-Reveron J, Tomasiewicz HC, Shetty A et al (2013) Stereotactic laser induced thermotherapy (LITT): a novel treatment for brain lesions regrowing after radiosurgery. J Neurooncol. https:// doi.org/10.1007/s11060-013-1142-2

14. Dindo D, Demartines N, Clavien PA (2004) Classification of surgical complications: A new proposal with evaluation in a cohort of 6336 patients and results of a survey. Ann Surg 240(2):205-213. https://doi.org/10.1097/01.sla.0000133083.54934.ae

15. Carai A, Mastronuzzi A, De Benedictis A et al (2017) Robotassisted stereotactic biopsy of diffuse intrinsic pontine glioma: a single-center experience. World Neurosurg. https://doi.org/10. 1016/j.wneu.2017.02.088

16. Minchev G, Kronreif G, Martínez-Moreno M et al (2017) A novel miniature robotic guidance device for stereotactic neurosurgical interventions: preliminary experience with the iSYS1 robot. J Neurosurg. https://doi.org/10.3171/2016.1.JNS152005

17. Li QH, Zamorano L, Pandya A et al (2002) The application accuracy of the NeuroMate robot - a quantitative comparison with frameless and frame-based surgical localization systems. Comput Aided Surg. https://doi.org/10.1002/igs.10035

18. González-Martínez J, Bulacio J, Thompson S et al (2016) Technique, results, and complications related to robot-assisted stereoelectroencephalography. Neurosurgery. https://doi.org/10.1227/ NEU.0000000000001034

19. Lefranc M, Le Gars D (2012) Robotic implantation of deep brain stimulation leads, assisted by intra-operative, flat-panel CT. Acta Neurochir (Wien). https://doi.org/10.1007/s00701-012-1445-7

20. Bjartmarz H, Rehncrona S (2007) Comparison of accuracy and precision between frame-based and frameless stereotactic navigation for deep brain stimulation electrode implantation. Stereotact Funct Neurosurg. https://doi.org/10.1159/000103262

21. Paleologos TS, Dorward NL, Wadley JP, Thomas DGT (2001) Clinical validation of true frameless stereotactic biopsy: Analysis of the first 125 consecutive cases. Neurosurgery. https://doi.org/ 10.1097/00006123-200110000-00009

22. Maciunas RJ, Galloway RL, Latimer JW (1994) The application accuracy of stereotactic frames. Neurosurgery. https://doi.org/10. 1227/00006123-199410000-00015

23. Sharma M, Rhiew R, Deogaonkar M et al (2014) Accuracy and precision of targeting using frameless stereotactic system in deep brain stimulator implantation surgery. Neurol India. https://doi. org/10.4103/0028-3886.144442

24. Barnett GH, Miller DW, Weisenberger J (1999) Frameless stereotaxy with scalp-applied fiducial markers for brain biopsy 
procedures: experience in 218 cases. J Neurosurg. https://doi.org/ 10.3171/jns.1999.91.4.0569

25. Bekelis K, Radwan TA, Desai A, Roberts DW (2012) Frameless robotically targeted stereotactic brain biopsy: feasibility, diagnostic yield, and safety - clinical article. J Neurosurg. https://doi.org/ 10.3171/2012.1.JNS111746

26. Owen CM, Linskey ME (2009) Frame-based stereotaxy in a frameless era: current capabilities, relative role, and the positive- and negative predictive values of blood through the needle. J Neurooncol. https://doi.org/10.1007/s11060-009-9871-y

27. Smith JS, Quiñones-Hinojosa A, Barbaro NM, McDermott MW (2005) Frame-based stereotactic biopsy remains an important diagnostic tool with distinct advantages over frameless stereotactic biopsy. J Neurooncol. https://doi.org/10.1007/s11060-004-4208-3

28. Fomenko A, Serletis D (2018) Robotic stereotaxy in cranial neurosurgery: a qualitative systematic review. Neurosurgery. https:// doi.org/10.1093/neuros/nyx576

29. Shakal AAS, Mokbel EAH (2014) Hemorrhage after stereotactic biopsy from intra-axial brain lesions: incidence and avoidance. $\mathrm{J}$ Neurol Surgery, Part A Cent Eur Neurosurg. https://doi.org/10. 1055/s-0032-1325633

30. Gralla J, Nimsky C, Buchfelder M et al (2003) Frameless stereotactic brain biopsy procedures using the stealth station: indications, accuracy and results. Zentralbl Neurochir 64(4):166-170. https://doi.org/10.1055/s-2003-44620

31. McGirt MJ, Woodworth GF, Coon AL et al (2005) Independent predictors of morbidity after image-guided stereotactic brain biopsy: a risk assessment of 270 cases. J
Neurosurg 102(5):897-901. https://doi.org/10.3171/jns.2005. 102.5.0897

32. Woodworth GF, McGirt MJ, Samdani A et al (2006) Frameless image-guided stereotactic brain biopsy procedure: diagnostic yield, surgical morbidity, and comparison with the frame-based technique. J Neurosurg 104(2):233-237. https://doi.org/10.3171/ jns.2006.104.2.233

33. Kim JE, Kim DG, Paek SH et al (2003) Stereotactic biopsy for intracranial lesions: reliability and its impact on the planning of treatment. Acta Neurochir (Wien). https://doi.org/10.1007/ s00701-003-0048-8

34. Brandmeir NJ, Savaliya S, Rohatgi P, Sather M (2018) The comparative accuracy of the ROSA stereotactic robot across a wide range of clinical applications and registration techniques. J Robot Surg. https://doi.org/10.1007/s11701-017-0712-2

35. Malham GM, Wells-Quinn T (2019) What should my hospital buy next?-Guidelines for the acquisition and application of imaging, navigation, and robotics for spine surgery. J Spine Surg 5(1):155165. https://doi.org/10.21037/jss.2019.02.04

36. Lefranc M, Peltier J (2016) Evaluation of the ROSA ${ }^{\mathrm{TM}}$ Spine robot for minimally invasive surgical procedures. Expert Rev Med Devices. https://doi.org/10.1080/17434440.2016.1236680

Publisher's Note Springer Nature remains neutral with regard to jurisdictional claims in published maps and institutional affiliations. 